\title{
The Extent of Polysynaptic Responses in the Dorsal Spinocerebellar Tract to Stimulation of Group I Afferent Fibers in Gastrocnemius-Soleus
}

\author{
C. E. Osborn and R. E. Poppele \\ Laboratory of Neurophysiology, University of Minnesota, Minneapolis, Minnesota 55455
}

\begin{abstract}
We investigated the extent of the distribution of polysynaptic connections to the dorsal spinocerebellar tract (DSCT). Recording from a randomly selected population of DSCT units, we found that over $60 \%$ responded to maximal stimulation of group I afferent fibers in the gastrocnemius-soleus muscle nerve. Most of the responses had a time course or latency consistent with polysynaptic activation. The extensive distribution of this input into the pool of DSCT neurons suggests that input from muscle nerves in general is widely distributed to these neurons. The results further imply that the DSCT plays a more integrative role in the transmission of information from the hindlimb to the cerebellum than has been previously supposed.
\end{abstract}

The dorsal spinocerebellar tract (DSCT) is a major source of information about the hindlimb for the cerebellum. Up to now, the organization of this tract has been examined primarily with respect to the monosynaptic activation of DSCT cells by peripheral receptors (Oscarsson, 1965; Mann, 1973; Bloedel and Courville, 1981). On the basis of these studies, it has been widely accepted that DSCT units transmit information from small, homogeneous groups of peripheral receptors (see, for example, Ito, 1984). This view implies that information received by the cerebellum via the DSCT is largely that provided by the peripheral receptors themselves.

It is implicit in this viewpoint that polysynaptic connections to the DSCT can be disregarded. However, earlier work suggests that polysynaptic input, cspecially of an inhibitory nature, can affect the output of DSCT neurons (Mann, 1973; Knox et al., 1977; Kubota and Poppele, 1977; Bloedel and Courville, 1981; Hongo et al., 1983a, b; Osborn and Poppele, 1983), but the extent of their distribution has never been studied systematically. Consequently, it is not known if this input comprises a minor aspect or a major component of DSCT activity.

This issue is explored in the present paper by describing the responses of a large sample of DSCT neurons to afferent fiber activity in the nerve to a single muscle group, gastrocnemiussoleus (GS). Using electrical activation of group I afferent fibers as a stimulus, we found that the activity of $64 \%$ of a sample of

Received Apr, 22, 1987; revised July 14, 1987; accepted July 21, 1987

We would like to thank Dr. C. A. Terzuolo for a critical reading of the manuscript. This work was supported in part by NIH Grants F32 NS 07147 and R01 NS 21143.

Correspondence should be addressed to C. E. Osborne, Department of Physiology 6-255 MH, 435 Delaware Street S.E., University of Minnesota, Minneapolis, MN 55455.

Copyright (C) 1988 Society for Neuroscience $0270-6474 / 88 / 010316-04 \$ 02.00 / 0$ randomly selected DSCT units was affected, predominantly by polysynaptic input. Such data suggest a widespread convergence from peripheral receptors onto DSCT cells. Consequently, the information provided to the cerebellum via the DSCT tract is likely to be more complex than that supplied from clusters of homogeneous receptors. A preliminary report of this study has been presented (Osborn and Poppele, 1984).

\section{Materials and Methods}

Single-unit recordings were made from spinal cords of adult cats, 2.5$5 \mathrm{~kg}$, deeply anesthetized with pentobarbital sodium (Nembutal; Park Davis), $35-40 \mathrm{mg} / \mathrm{kg}$, i.p., initially, supplemented by intravenous administration during the experiment as the animal became reflexly active to maintain a surgical anesthetic state. The responses from 152 identified axons of the DSCT at the $T_{12}$ and $T_{11}$ spinal cord levels to group I electrical stimulation of the GS nerve were recorded in 12 cats. The surgical and recording procedures have been described previously (Knox et al., 1977), but pertinent details are given below.

The left hindlimb was denervated and the left ventral roots $S_{1}$ and $\mathrm{L}$, were cut at least $1 / 2 \mathrm{hr}$ prior to unit recording to insure that injury potentials did not contribute to the results. A recording electrode on the $S_{1}$ dorsal root monitored the incoming afferent volley. A Poissondistributed pulse train having a mean rate of $8 / \mathrm{sec}$ was arbitrarily chosen as the stimulus (see Knox and Poppele, 1977, for its rationale in determining cross-correlograms). Stimulus intensity was held at $2 \times$ threshold for the group I dorsal root volley to maximally activate Ia and $\mathrm{Ib}$ fibers, although some of the group II fibers were probably excited as well (Brock et al., 1951). An electrode placed in the inferior peduncle of the cerebellum was used to activate DSCT neurons antidromically.

Action potentials from spontaneously active DSCT units were recorded from their axons in the dorsolateral funiculus using glass-coated tungsten microelectrodes ( $2-4 \mathrm{M} \Omega$; Frederick Haer). Units were identified as belonging to the DSCT by their response to antidromic activation from the ipsilateral inferior peduncle and by the resetting of their spontaneous background discharge following the antidromic spike (Knox et al., 1977). Action potentials from DSCT units were converted to pulse trains by an analog-to-digital converter, and their times of occurrence were stored on disk, along with the occurrence times of the input pulse train, for off-line analysis.

The effect of a stimulus on the discharge of a single unit was determined from the difference between the waiting time and forward-recurrence time probability density functions (Gerstein, 1970). The waiting time density was calculated as the density of intervals between any stimulus and the first DSCT spike. The forward-recurrence time density is an equivalent function calculated without reference to a stimulus, and may be approximated from the interspike interval density (Cox and Lewis, 1966). Any difference between these functions can be taken as evidence of an effect of the stimulus on the spike train. The KolmogorovSmirnov test (Lindgren, 1968) was used to this end. If the test failed, i.e., if the distributions were significantly different, we assumed that the difference was caused by the incoming volley.

\section{Results}

We recorded impulse activity from 152 spontaneously firing units identified as belonging to the DSCT (see Materials and 
Table 1. Summary of responses to group I stimulation of GS: percentage of units responding and each response type, and comparison with grouping according to conduction velocity computed from antidromic latency

\begin{tabular}{|c|c|c|c|c|c|c|c|c|}
\hline & \multicolumn{4}{|c|}{ Total trials } & \multicolumn{4}{|c|}{$\underline{\text { Significant responses }}$} \\
\hline & No. & $\%^{a}$ & No. & $\%^{b}$ & $5 \%{ }^{a}$ & $1 \% \%^{b}$ & $\begin{array}{l}<70 \\
\mathrm{~m} / \mathrm{sec}\end{array}$ & $\begin{array}{l}>70 \\
\mathrm{~m} / \mathrm{sec}\end{array}$ \\
\hline $\begin{array}{l}\text { Total significant } \\
\text { Excitatory }\end{array}$ & 100 & 64 & 81 & 52 & $64 \%$ & $52 \%$ & $64 \%$ & $60 \%$ \\
\hline Short latency & 19 & 12 & 18 & 12 & $19 \%$ & $22 \%$ & $21 \%$ & $17 \%$ \\
\hline Long latency & 21 & 13 & 16 & 10 & $21 \%$ & $20 \%$ & $29 \%$ & $17 \%$ \\
\hline Inhibitory & 60 & 38 & 47 & 30 & $60 \%$ & $58 \%$ & $50 \%$ & $67 \%$ \\
\hline No response & 57 & 36 & 74 & 47 & & & & \\
\hline Total trials & 157 & 100 & 155 & 100 & & & & \\
\hline
\end{tabular}

${ }^{a}$ Response significance at the $5 \%$ level.

${ }^{b}$ Response significance at the $1 \%$ level.

Methods). Electrode penetrations were made at random into the dorsolateral funiculus at $\mathrm{T}_{11}-\mathrm{T}_{12}$ spinal levels. Axons were found throughout the entire funiculus to a depth of 2-3 mm (Loewy, 1970). Conduction velocities determined from antidromic spike latencies ranged from 30 to $118 \mathrm{~m} / \mathrm{sec}$ (mode, $70 \mathrm{~m} / \mathrm{sec}$ ). The spontaneous discharge rate ranged from 3 to $48 \mathrm{impulses} / \mathrm{sec}$ (mean, $17.4 \pm 11 \mathrm{impulses} / \mathrm{sec}$ ). Interval distributions were generally the same as those reported previously (Knox et al., 1977).

We recorded from every DSCT unit that could be isolated regardless of whether or not the unit appeared to respond to the stimulus to the GS nerve. For each trial the presence or absence of a response was established by the Kolmogorov-Smirnov test for differences in distribution functions at the 0.05 level (see Materials and Methods). Under these conditions of random sampling over a large population, $64 \pm 8 \%^{1}$ of the units responded in a statistically significant manner. Even when the criterion for significance was raised to the 0.01 level, more than half of the units were found to respond in each experiment; see Tables 1 and 2 . The tables also show the percentage of responsive units present in subsets of units, including units with conduction velocities above or below the mode.

Responses accepted as significant on the basis of statistical criteria were generally obvious from visual inspection of the cross-correlograms (Fig. 1). These were qualitatively similar to those reported earlier (Knox et al., 1977). Nearly all responses $(81 \%)$ were consistent with polysynaptic activation, either longlasting inhibition (60\%; type 2 of Knox et al., 1977) or late excitation, i.e., a peak occurring more than $10 \mathrm{msec}$ after the stimulus $(21 \%$; type 3$)$. Only about half of all excitatory responses had a peak within $10 \mathrm{msec}$ of the stimulus, suggesting a contribution by a monosynaptic input. These results are summarized in Tables 1 and 2, where it can also be seen that the percentage of inhibitory and excitatory responses was consistent across samples of units recorded in different experiments and was independent of the statistical criterion $(0.05 \mathrm{vs} 0.01)$ used in assessing the presence or absence of a response.

Although it was usually necessary to average the response to several hundred stimuli to ascertain the presence of a response in a single unit, the presence of a given type of response to a

\footnotetext{
${ }^{1}$ The standard deviation was estimated by assuming a random sample ( $\mathrm{SD}=$ $1 / \sqrt{n}$, where $n=$ total number of units in the sample (Lindgren, 1968). The implied a verage error of $5 \%$ in estimating response significance was not included. Note, however, the result of determining significance at the $1 \%$ level (Table 1 ).
}

single stimulus could often be appreciated from the average spike density across a population of units with similar behavior (Figs. 2, 3). The raster plot of Figure 2 depicts the spike activity of individual DSCT units following a stimulus applied to GS (vertical line). The units are representative of the total sample, and are grouped according to the type of response.

A more quantitative measure is presented in Figure 3 , in which the raster plot for each response type is plotted in a separate panel, along with a peristimulus time histogram (PSTH) computed across units. It is clear from the PSTH that the density of spikes in the cell population is significantly altered from its background (prestimulus) level for $50 \mathrm{msec}$ or more following a single stimulus, even though any given unit may have only a weak response to a particular stimulus. In fact, the population density is similar to the poststimulus density for single units averaged over time (see cross-correlograms of Fig. 1). Thus, the effect of a particular input volley on the DSCT is manifested by the size of the population of DSCT units affected by the stimulus rather than in the magnitude of the responses of individual units.

\section{Discussion}

We found that over $60 \%$ of a large, randomly selected sample of DSCT units responds to stimulation of the nerve to a single muscle group at $2 \times$ threshold for group I fibers, which implies that there is an extensive divergence of input from a single muscle group into the pool of DSCT neurons. This conclusion rests on the representative nature and the adequacy of the experimental sample. Although the sample is small compared to the total number of fibers in the tract (7000-10,000; Loewy,

Table 2. Subgroups of response data: 3 nonoverlapping sets of 50 units, each set from 4 cats, with different cats in each group

\begin{tabular}{|c|c|c|c|c|c|c|}
\hline & \multicolumn{2}{|c|}{ Group A } & \multicolumn{2}{|c|}{ Group B } & \multicolumn{2}{|c|}{ Group C } \\
\hline & No. & $\%$ & No. & $\%$ & No. & $\%$ \\
\hline Total significant & 34 & 68 & 27 & 54 & 30 & 60 \\
\hline \multicolumn{7}{|l|}{ Excitatory } \\
\hline Short latency & 5 & 10 & 6 & 12 & 5 & 10 \\
\hline Long latency & 7 & 14 & 4 & 8 & 7 & 14 \\
\hline Inhibitory & 22 & 44 & 17 & 34 & 18 & 36 \\
\hline No response & 16 & 32 & 23 & 46 & 20 & 40 \\
\hline Total trials & 50 & 100 & 50 & 100 & 50 & 100 \\
\hline
\end{tabular}




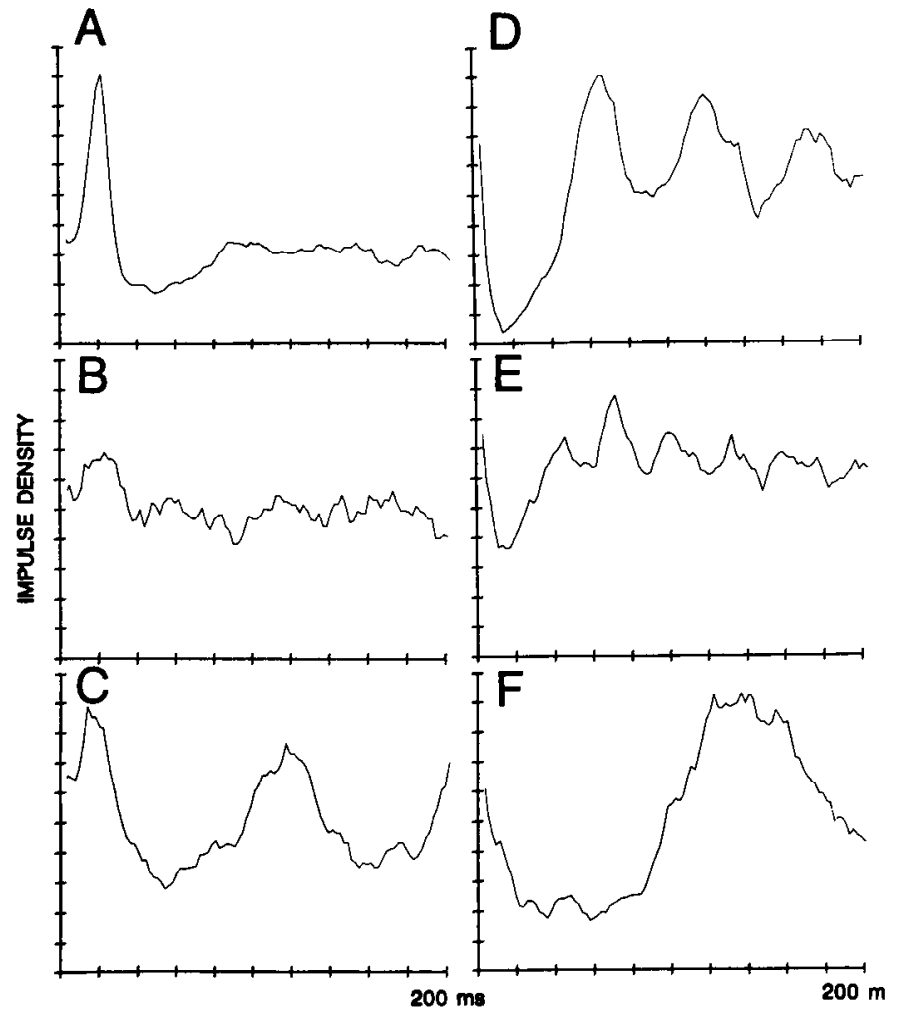

Figure 1. Cross-correlograms of individual DSCT units. Sample crosscorrelograms representing time averages of responses to 300-500 stimuli in each case; $3 \mathrm{msec}$ bin width. Stimuli presented at random (Poissondistributed, mean rate $8 / \mathrm{sec}$ ) were $2 \times$ threshold for activation of group I afferent fibers in GS. $A-C$, Three excitatory responses. $D-F$, Three inhibitory responses.

1970 ), the probability that less than $50 \%$ of the DSCT units receives input from GS is remote $(p<0.0015$, assuming a binomial distribution) if the units were selected at random and no systematic bias was present in the experimental protocol.

In this context, it should be stressed that no bias was intentionally introduced in selecting the sample population except for that imposed by the requirement for spontaneous activity. Indeed, we found fibers throughout the entire dorsolateral funiculus, using antidromic invasion as the sole criterion for unit identification. Spontaneous background activity was required in order to observe inhibitory responses and to apply the test for statistical significance. However, nearly all DSCT units are generally active (Mann, 1971), except for a minor group of exteroceptive units that are silent in the absence of adequate stimulation. We encountered very few DSCT units that were not spontaneously active, and no more than we encountered in similar preparations with the hindlimb nerves intact (Osborn and Poppele, 1984, and unpublished observation).

A different type of bias may have been introduced by the recording electrodes, which could have preferentially selected large-diameter axons. Usually the extent of such bias is measured by comparing the distribution of conduction velocities with that computed from the fiber-diameter spectrum, but for the DSCT no such data on fiber diameters are available. Any underrepresentation of small-diameter fibers could bias the sample if the response parameters are correlated with axon diameter or conduction velocity. This was dealt with in an earlier study, which found no evidence for a relationship between the

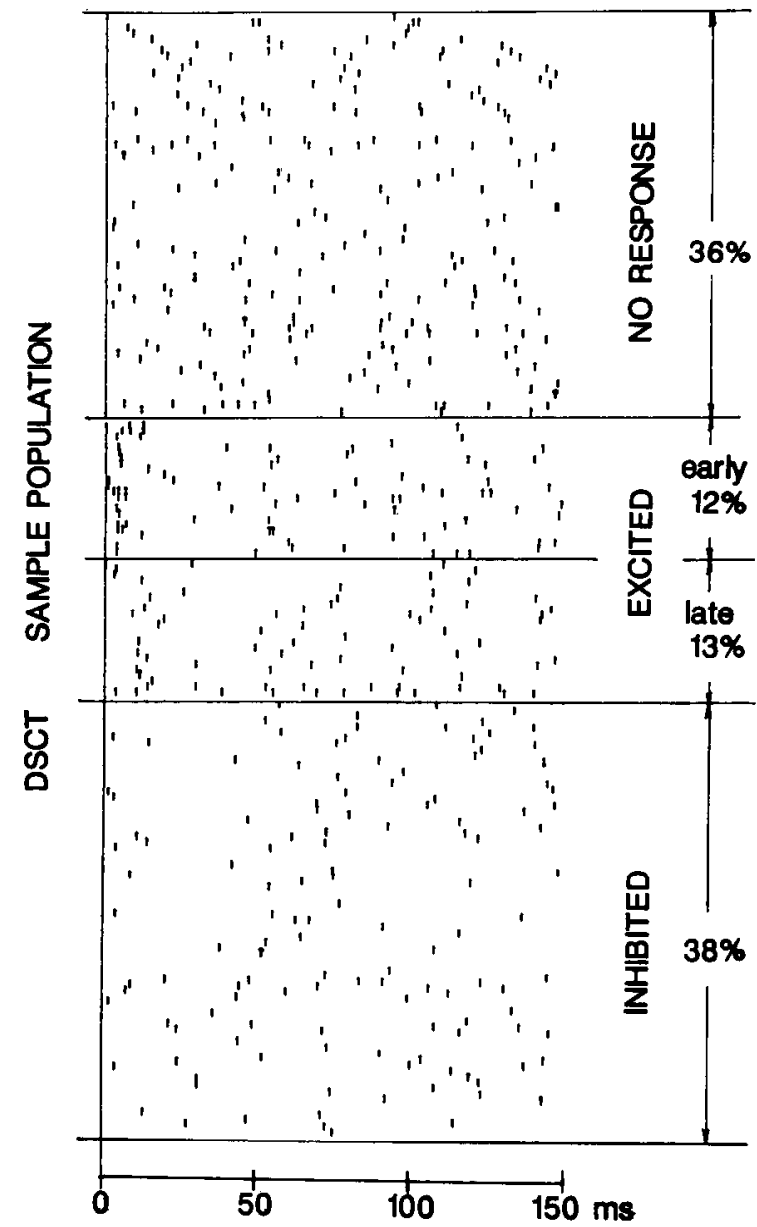

Figure 2. Effect of the stimulus on DSCT activity. Raster diagram of the activity in 100 DSCT units (2 raster lines/unit) selected at random from the DSCT. Activity is lined up with the presentation of a single stimulus to the GS nerve at $2 \times$ threshold for group I activation. The presence or absence of a response in each case was determined statistically from records of no fewer than 900 spikes (see Materials and Methods for description of statistical test). Segments were chosen for this illustration using the sole criterion that no stimulus was presented for at least $500 \mathrm{msec}$ prior to the stimulus marking the beginning of each record. The upper group gave no detectable response. The activity in the middle group was accelcratcd by the stimulus, and that in the lower group was slowed. The number of units in each group is in proportion to the percentage of units in the population responding in a similar manner. The illustration therefore represents the alteration of activity across units of the tract by a single shock to a single peripheral muscle nerve.

antidromic latency of a unit and either its response to nerve stimulation or the parameters of its spontaneous activity (Knox et al., 1977). In this study we found no difference in the percentage of units responding to GS stimulation based on conduction velocity, although there may have been less inhibition of the faster conducting cells.

Finally, differences in experimental conditions appear to have had little influence on the results of this study. Three subsets of data taken from different preparations were found to behave similarly in terms of percentage of units affected by the stimulus.

We can therefore conclude that the responses we have described are representative of the DSCT activity at $T_{12}$, and further suggest that a wide divergence of input from muscle nerve onto DSCT units is a general feature of such activity. This conclusion is also consistent with the data from both anatomical 
A

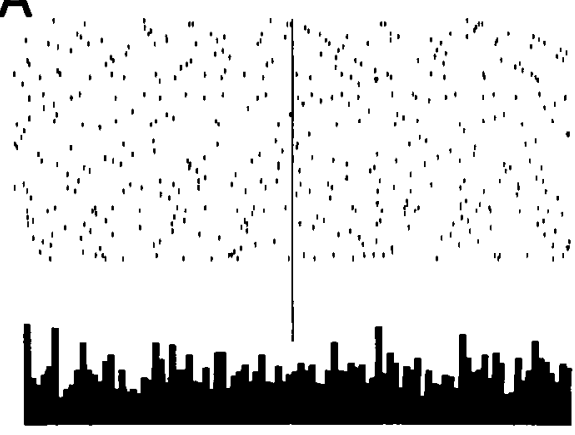

B

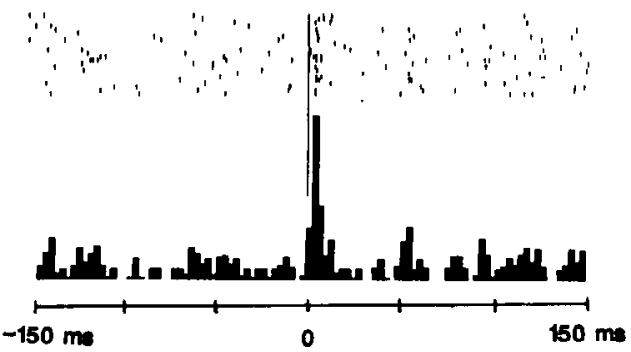

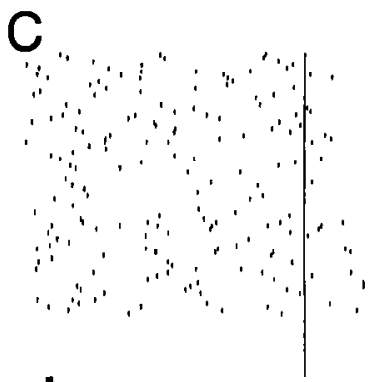

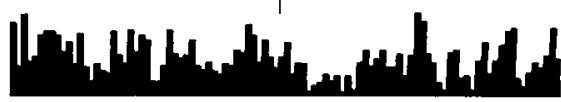

D

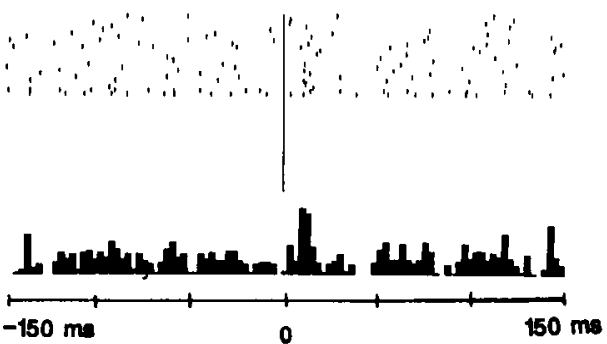

Figure 3. Average response of groups of DSCT units. The same rasters of Figure 2 replotted with peristimulus time histograms of spike density across units for each response group. $A$, No response. $B$. Excitation peak prior to 10 msec. $D$, Excitation peak later than at $10 \mathrm{mscc}$. $C$, Inhibitory responses. and physiological studies of DSCT, which have provided evidence for both monosynaptic and polysynaptic inputs to DSCT cells. However, the extent of such responses has not previously been documented.

The divergence of input from muscle nerves implied by these results shows that the information carried by the DSCT is not discretely organized muscle by muscle. This is in contrast with the currently prevailing view that each DSCT unit acts essentially as a relay between sensory receptors and the cerebellum, with negligible central processing by DSCT neurons. This proposition is likely to have arisen because of the emphasis placed on monosynaptic connections. While the data presented here do not dispute the presence of a restricted distribution of monosynaptic components, the presence of a widely divergent polysynaptic component lends an entirely different perspective to the way information from the limbs may be encoded by cerebellar afferents. It is a perspective that is more consonant with both current hypotheses about other cerebellar afferent systems (Wilson and Peterson, 1981) and with the increasing evidence of complexity of interneuronal circuits of the spinal cord (Hongo et al., 1983a, b). The role of integrating afferent information from the various muscles and joints of the limb no longer seems to be the exclusive province of the cerebellum, but also appears to be shared by spinal centers such as the DSCT.

\section{References}

Bloedel, J. R., and J. Courville (1981) Cerebellar afferent systems. In Handbook of Physiology, 1: The Nervous System, vol. II: Motor Control, J. M. Brookhart, V. B. Mountcastle, and S. R. Geiger, eds., pp. 735-829, American Physiological Society, Bethesda, MD.

Brock, L. G., J. C. Eccles, and W. Rall (1951) Experimental investigations on the afferent fibres in muscle nerves. Proc. R. Soc. Lond. [Biol.] 138: 453-475.

Cox, D. R., and P. A. W. Lewis (1966) The Statistical Analysis of Series of Events, Wiley, New York.

Gerstein, G. L. (1970) Functional association of neurons: Detection and interpretation. In Neuroscience: Second Study Program, F. O. Schmitt, ed., pp. 648-661, Rockefeller U. P., New York.

Hongo, T., E. Jankowska, T. Ohno, S. Saski, M. Yamashita, and K. Yoshida (1983a) Inhibition of dorsal spinocerebellar tract cells by interneurones in upper and lower lumbar segments in the cat. J. Physiol. (Lond.) 342: 145-149.

Hongo, T., E. Jankowska, T. Ohno, S. Saski, M. Yamashita, and K. Yoshida (1983b) The same interneurones mediate inhibition of dorsal spinocerebellar tract cells and lumbar motoneurones in the cat. J. Physiol. (Lond.) 342: 161-180.

Ito, M. (1984) The Cerebellum and Neural Control, Raven, New York, pp. 206-209.

Knox, C. K., and R. E. Poppele (1977) Correlation analysis of stimulus-evoked changes in excitability of spontaneously firing neurons. J. Neurophysiol. 40: 616-626.

Knox, C. K., S. Kubota, and R. E. Poppele (1977) A determination of excitability changes in dorsal spinocerebellar tract neurons from spike-train analysis. J. Neurophysiol. 40: 626-646.

Kubota, S., and R. E. Poppele (1977) Evidence for control of DSCT activity by the brain stem reticular formation. Brain Res. 129: 361365.

Lindgren, B. W. (1968) Statistical Theory, Macmillan, New York.

Loewy, A. (1970) A study of neuronal types in Clarke's column in the adult cat. J. Comp. Neurol. 139: 53-80.

Mann, M. D. (1971) Axons of dorsal spino-cerebellar tract which respond to activity in cutaneous receptors. J. Neurophysiol. 34: 10351050.

Mann, M. D. (1973) Clarke's column and the dorsal spinocerebellar tract: A review. Brain Behav. Evol. 7: 34-83.

Osborn, C. E., and R. E. Poppele (1983) Cross-correlation analysis of the response of units in the dorsal spinocerebellar tract (DSCT) to muscle stretch and contraction. Brain Res. 280: 339-342.

Osborn, C. E., and R. E. Poppele (1984) Responses of populations of DSCT neurons to natural stimulation. Soc. Neurosci. Abstr. 10: 744.

Oscarsson, O. (1965) Functional organization of the spino- and cuneocerebellar tracts. Physiol. Rev. 45: 495-522.

Wilson, V. J., and B. W. Peterson (1981) Vestibulospinal and reticulospinal systems. In Handbook of Physiology, 1: The Nervous System. vol. II: Motor Control, J. M. Brookhart, V. B. Mountcastle, and S. R. Geiger, eds., pp. 667-702, American Physiological Society, Bethesda, MD. 